\title{
RECAUDACIÓN DE IMPUESTOS
}

\section{TUMEUBAHUA}

Los impuestos son los pagos o prestaciones que se entregan al Gobierno de acuerdo al mandato legal, con el objetivo de satisfacer las necesidades comunes de todos los ciudadanos.

Existen dos tipos de impuestos, los directos aquellos impuestos que gravan o afectan directamente lo que se recibe como ingreso por ejemplo el Impuesto a la Renta; e, indirectos aquellos que gravan o afectan a lo que se consume o se gasta, como el Impuesto al Valor Agregado IVA. Todos estos Impuestos son utilizados por el Gobierno para cubrir parte del Gasto Público, que se invierte en educación, salud, seguridad, infraestructura vial, entre otros aportes para el desarrollo del país.

A continuación se presenta información estadística respecto a la recaudación tributaria a nivel nacional, según datos del Servicio de Rentas Internas.

RECAUDACIÓN EFECTIVA

\begin{tabular}{|c|c|c|c|c|c|c|c|}
\hline PROIIIAIAS & Enaro & Fehnreno & Marizo & Ahrill & Miajo & Junio & Total \\
\hline AZUAY & $6,583,897.94$ & $8,448,098.46$ & $33,397,693.73$ & $51,972,502.67$ & $1,658,513.00$ & $0,377,875.38$ & 222,4 \\
\hline BOLIVAR & $889,258.77$ & $649,717.46$ & $1,426,449.17$ & $1,508,516.18$ & $901,948.74$ & $886,690.73$ & 58105 \\
\hline CAÑAR & $2,787,492.72$ & $1,819,424.71$ & $2,681,619.41$ & $7,107,339.42$ & $2,402,568.96$ & $1,898,441.70$ & 886.91 \\
\hline CARCHI & $2,159,838.20$ & $1,502,675.92$ & 8.75 & $2,080,152.71$ & $1,891,836.22$ & $1,560,587.47$ & 259.27 \\
\hline $\mathrm{CHIMBO}$ & .57 & 7.39 & 2.45 & $5,457,4$ & $4,637,7$ & 1,482, & 710.75 \\
\hline PAXI & 28 & 6.37 & 5,220 & 5,726 , & $3,845,7$ & $4,653,5$ & 172.26 \\
\hline EL ORO & 11,101 & 6.14 & 16,592, & 21,767 & $12,953,7$ & $12,061,705.02$ & 736.54 \\
\hline ESMERALDAS & 29 & 6.71 & 4.017 & 4,600 & $4,167,30$ & 3,934 & ,881.22 \\
\hline GALAPAGOS & 1,987, & 1,247, & 1,267, & 2,798 & $2,364,0$ & $1,307,959.40$ & 192.95 \\
\hline GUAYAS & $98,517,7$ & $10,776,155.41$ & $233,893,440.27$ & $484,384,3$ & $229,188,303.73$ & $225,076,961.16$ & ,989.29 \\
\hline IMBABURA & $8,291,848.83$ & $5,882,283.11$ & $7,691,329.98$ & $20,452,47$ & $6,809,345.25$ & $5,617,417.33$ & ,701.52 \\
\hline \begin{tabular}{|l} 
LOJA \\
\end{tabular} & $6,430,68$ & $4,092,872.53$ & $6,470,056.58$ & $6,475,5$ & $4,550,42$ & $4,685,335.72$ & 903.72 \\
\hline LOS RIOS & 4,644, & $5,075,246.10$ & 4,635 & $5,066,2$ & $4,747,32$ & $4,695,806.80$ & 802.47 \\
\hline MANABI & $19,065,4$ & $13,377,500.31$ & $15,607,912.17$ & $21,076,88$ & $16,647,830.68$ & $16,356,274.10$ & 815.35 \\
\hline MORONA SANTIAGO & $1,186,255.11$ & $979,788.64$ & $1,447,406.71$ & $1,134,7$ & $1,291,167.59$ & $994,654.18$ & 037.26 \\
\hline NAPO & $1,336,5$ & $717,387.09$ & $960,814.11$ & $1,349,2$ & $1,082,802.66$ & $893,327.98$ & 122.86 \\
\hline ORELLANA & $2,506,55$ & $2,882,360.43$ & $2,852,0$ & $2,515,0$ & $2,432,4$ & $2,513,535.60$ & $15,701,966.85$ \\
\hline PASTAZA & $1,180,6$ & $782,090.43$ & $967,384.83$ & $1,145,4$ & 946,3 & $995,600.21$ & $6,017,472.30$ \\
\hline PICHINCHA & $45,454,8$ & $20,943,776.27$ & $444,496,3$ & $50,549,6$ & $453,391,4$ & $432,585,807.05$ & 961.48 \\
\hline SANTA ELENA & 2,123 & $1,608,322.84$ & $2,567,20$ & $2,037,9$ & $2,083,0$ & $1,916,876.40$ & 117.05 \\
\hline $\begin{array}{l}\text { SANTO DOMINGO } \\
\text { DE LOS TSACHILAS }\end{array}$ & $5,522,7$ & $4,363,131.07$ & $4,369,66$ & $4,785,3$ & $3,941,8$ & $4,297,758.68$ & 522.06 \\
\hline SUCUMBIOS & 62 & .10 & 2.55 & 81 & 64 & .69 & 601.41 \\
\hline TUNGURAHUA & $12,631,830.72$ & $8,780,014.17$ & $12,654,105.44$ & $14,905,6$ & $11,656,675.11$ & $9,541,616.75$ & $70,169,939.59$ \\
\hline ZAMORA CHINCl & $1,451,420.29$ & $921,442.29$ & $2,878,444.60$ & $1,611,199.55$ & $1,096,528.60$ & $1,226,096.73$ & $9,185,132.06$ \\
\hline NO ASIGNADOS & $4,673,535.73$ & $6,680,267.46$ & $11,714,038.52$ & $6,406,780.84$ & $5,525,376.91$ & $5,480,614.10$ & $40,480,613.56$ \\
\hline Total general & $1,097,074,598.22$ & $743,478,314.55$ & $826,806,340.23$ & $1,529,300,051.85$ & $812,622,930.95$ & $781,553,465.18$ & $5,790,835,700.98$ \\
\hline
\end{tabular}


En la tabla 1, se puede observar la recaudación a nivel Nacional desde enero a junio de 2014, donde se detalla las provincias y se puede evidenciar cuales son las que tienen mayor recaudación de impuestos en nuestros país durante el periodo especificado.

\section{Figura1. Aporte de las Provincias a la Recaudación Nacional de Impuestos}

Enero - Junio 2014

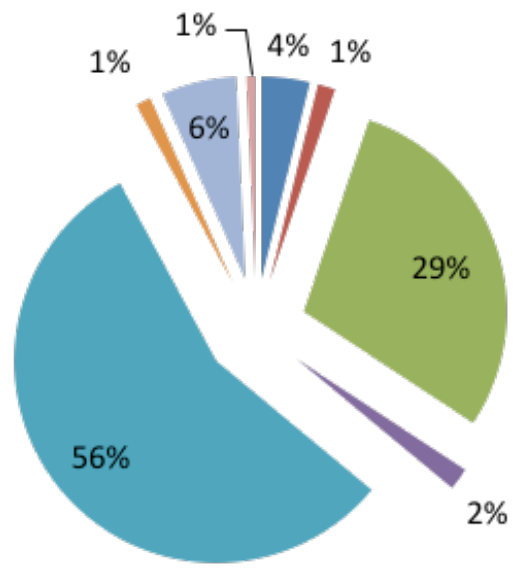

AZUAY
EL ORO
GUAYAS
MANABI
PICHINCHA
TUNGURAHUA
RESTO DE PROVINCIAS
NO ASIGNADOS

Fuente: Servicio de Rentas Internas S.R.I.

Elaboración: Equipo Técnico OBEST.

En el Gráfico 1, se determina que Pichincha aporta a la recaudación Nacional con un 56,08\% es decir con 3,247,421,961.48 USD., equivale a más de la mitad de lo que recaudan las 23 provincias restantes, seguida Guayas con 29,04\%, Azuay 3.84\%, Manabí $1,76 \%$, El Oro 1,45\%, Tungurahua 1,11\%, y las otras 19 provincias se distribuyen el 6,61\%, siendo Pastaza con menor recaudación apenas el 0,10\%. Tungurahua se encuentra en la posición 7 en recaudación de impuestos a nivel Nacional.

\section{Figura 2. Recaudación de Impuestos en Tungurahua}

Primer semestre del 2013 y 2014

US\$ dólares

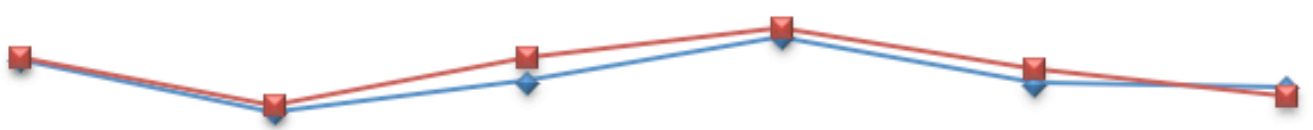

\begin{tabular}{|c|c|c|c|c|c|c|}
\cline { 2 - 7 } \multicolumn{1}{c|}{} & Enero & Febrero & Marzo & Abril & Mayo & Junio \\
\hline$\sim-2013$ & $12.391 .561,67$ & $8.294 .783,40$ & $10.724 .435,48$ & $14.152 .520,34$ & $10.469 .625,44$ & $10.194 .843,85$ \\
\hline$\longrightarrow-2014$ & $12.631 .830,72$ & $8.780 .014,17$ & $12.654 .105,44$ & $14.905 .697,40$ & $11.656 .675,11$ & $9.541 .616,75$ \\
\hline
\end{tabular}







En la Tabla 2, se detallan los impuestos recaudados en la Provincia de Tungurahua en sus 9 Cantones, para determinar cual genera mayor nivel de recaudación a nivel provincial.

Figura 3. Aporte de los Cantones a la Recaudación de la Provincia de Tungurahua Enero - Junio 2014

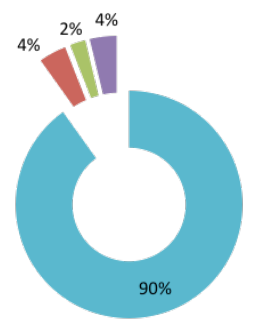

AMBATO

- PELILEO

BAÑOS

- RESTO DE CANTONES

Fuente: Servicio de Rentas Internas S.R.I.

Elaboración: Equipo Técnico OBEST.

En Tungurahua el cantón que más contribuye a la recaudación de impuestos es Ambato una ciudad comercial y de producción entre otras actividades, aporta con $90,1 \%$, seguido de Pelileo ciudad dedicada a la confesión del jean, fabricación de muebles y a la avicultura con 3,9\%, Baños ciudad turística 2,2\%, mientras que los demás cantones se dividen el porcentaje restante $3,8 \%$.

El mayor generador de impuesto en Ambato, es el Impuesto al Valor Agregado que aporta con el 43\% representa 2191420.98 USD a la recaudación total en la ciudad, a continuación le sigue Retenciones en la fuente $25,30 \%$, Renta de las Sociedades $8,2 \%$, Renta a las Personas Naturales 7,6\%, Impuesto a los Vehículos Motorizados Internos 5,4\%, Impuesto Ambiental a la Contaminación Vehicular 3.4\%, Impuestos a Consumos

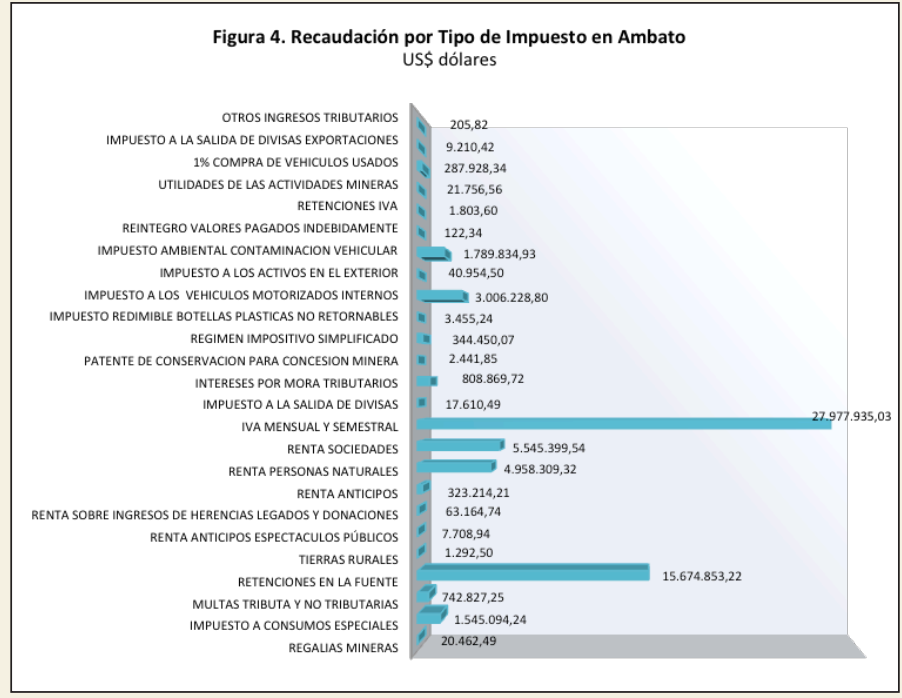

Fuente: Servicio de Rentas Internas S.R.I. Elaboración: Equipo Técnico OBEST.

Especiales con 2,4\%, Multas tributarias y no tributarias 1,4\%, Intereses por Mora Tributaria 1,2\% y finalmente los demás impuestos están bajo el 1\% de aportación al total de recaudación en el Cantón Ambato.

\section{Referencias}

$>\quad$ Servicio de Rentas Internas S.R.I. Estadísticas (En Línea). Observatorio Económico y Social de Tungurahua (OBEST) - Universidad Técnica de Ambato.

Econ. Tatiana Vayas

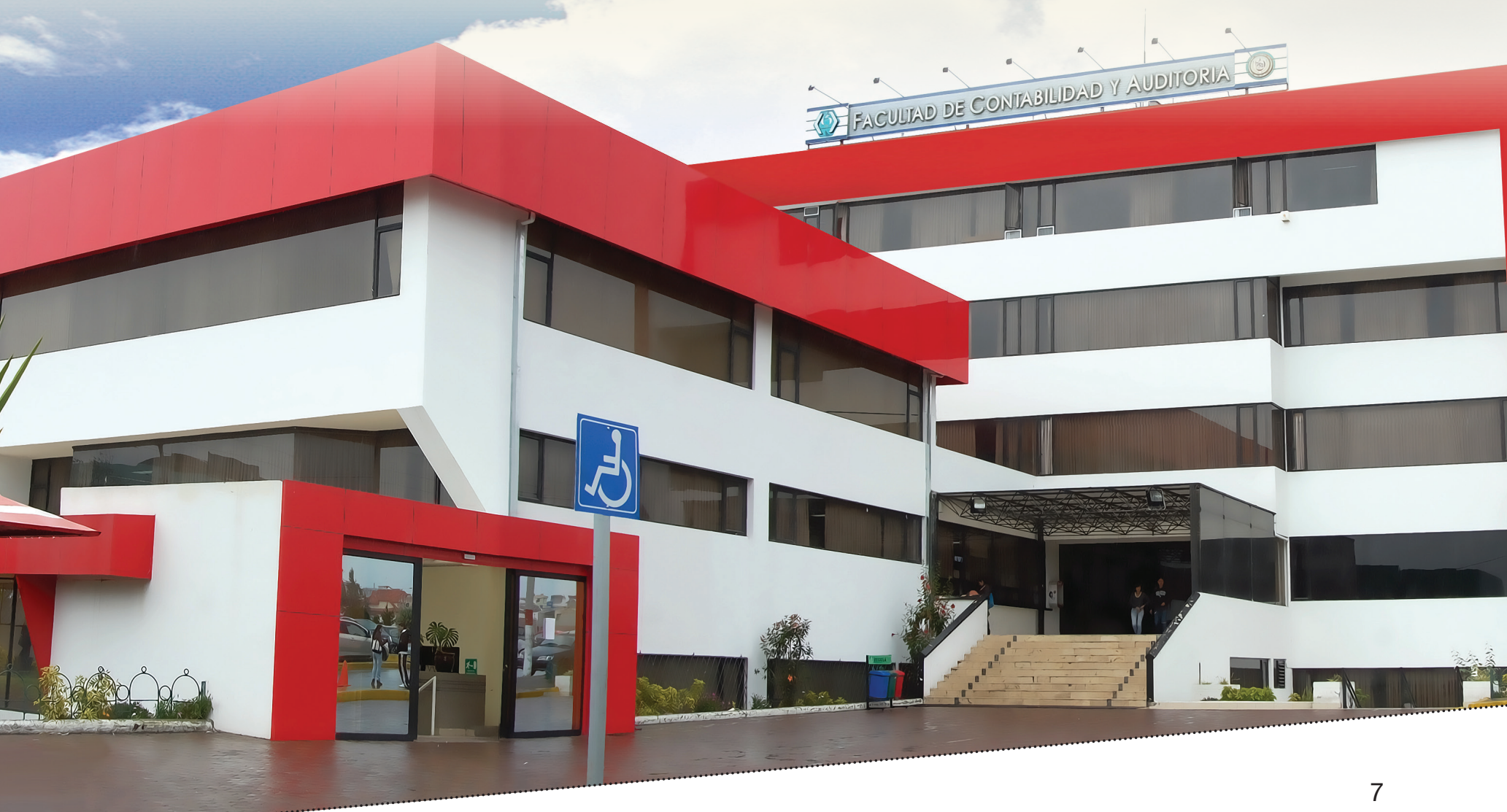

